\begin{tabular}{|c|c|}
\hline Citation & $\begin{array}{l}\text { Yang Zhang, Dixian Zhao, Patrick Reynaert, (2016), } \\
\text { A Flip-Chip Packaging Design With Waveguide Output on Single-Layer } \\
\text { Alumina Board for E-Band Applications } \\
\text { 1255-1264. }\end{array}$ \\
\hline Archived version & $\begin{array}{l}\text { Author manuscript: the content is identical to the content of the published } \\
\text { paper, but without the final typesetting by the publisher }\end{array}$ \\
\hline Published version & http://ieeexplore.ieee.org/stamp/stamp.jsp?tp=\&arnumber=7433474\&tag=1 \\
\hline Journal homepage & https://www.mtt.org/ \\
\hline Author contact & $\begin{array}{l}\text { Yang.zhang@esat.kuleuven.be } \\
\text { +3216374756 }\end{array}$ \\
\hline
\end{tabular}

(article begins on next page) 


\title{
A Flip-Chip Packaging Design with Waveguide Output on Single-Layer Alumina Board for E-Band Applications
}

\author{
Yang Zhang, Student Member, IEEE, Dixian Zhao, Member, IEEE, Patrick Reynaert, Senior Member, IEEE
}

\begin{abstract}
This paper presents a millimeter-wave packaging design for E-band long-haul point-to-point communications. A broadband flip-chip interconnect with standard $75 \mu \mathrm{m}$ pad pitch is developed on alumina substrate. To meet the application requirement, a microstrip line to $W R-12$ waveguide transition is proposed. The transition has no back-short cavity or modified waveguide involved. A back-to-back transition measurement is conducted to characterize the performance. Results show that the working bandwidth is from 69.5 to $87 \mathrm{GHz}$ and the insertion loss at $77 \mathrm{GHz}$ is less than $0.5 \mathrm{~dB}$. A 40-nm CMOS power amplifier is packaged and measured with the proposed packaging design. The entire packaging loss is only $2.5 \mathrm{~dB}$ from pads to waveguide, including the loss of a $10 \mathrm{~mm}$ microstrip line. The simulated and measured results are in good agreement. The gain and peak output power are $13.4 \mathrm{~dB}$ and $17.6 \mathrm{dBm}$. The silicon temperature is measured as $44.4^{\circ} \mathrm{C}$ after saturated output for 30 minutes. The performance is close to probe measurement after deembedding the packaging loss. Comparison between bondwire and flip-chip packaging is discussed and both are verified in millimeter-wave packaging design.
\end{abstract}

Index Terms-millimeter-wave, flip-chip, rectangular waveguide, wideband transition, power amplifier, CMOS, E-band, packaging

\section{INTRODUCTION}

A PPLICATIONS in millimeter-wave (mm-wave) range, such as high data rate personal area networks $(60 \mathrm{GHz})$, automobile radar systems $(77 \mathrm{GHz})$, high resolution imaging systems $(94 \mathrm{GHz})$, and E-band long-haul point-to-point communications (71-76 and 81-86 GHz) have drawn great attention in recent years [1]-[5]. Advanced CMOS technology manifests itself in high yield, high integration density, and low cost in production volume, which are essential to the population of realizing compact commercial systems for these (mm-wave) application areas [6]-[10]. To implement fully functional system integration, system-on-chip (SoC) is widely chosen, as it avoids the lossy interconnect. However, on-chip passive components such as antennas are still bulky in (mmwave) range, and suffer from performance deterioration caused by the lossy Silicon substrate and inter-metal-layer space limitation. On the other hand, system-in-package $(\mathrm{SiP})$ is a more appropriate approach in (mm-wave) applications. Instead of building everything on a single die, bulky components

Yang Zhang and Patrick Reynaert are with the Department of Electrical Engineering, Micro-Electronics and Sensors (ESAT-MICAS), University of Leuven, B-3001 Leuven, Belgium (e-mail: yang.zhang@esat.kuleuven.be; patrick.reynaert@esat.kuleuven.be).

Dixian Zhao is with the National Mobile Communication Research Laboratory, Southeast University, Nanjing, China (e-mail: dixian.zhao@seu.edu.cn). and functional units are designed on different carriers, thus flexibilities are introduced into design and high performance could be achieved. Among the solutions available, embedded wafer level packaging and embedded wafer level ball grid array (eWLB) are attractive for (mm-wave) applications [11]-[13]. They enable high level system integration when numerous I/O pins are used. The high integration is achieved by utilizing redistribution layers (RDLs) and through silicon vias (TSVs). However, the RF signal interconnections still remain challenging. RF signals are quite sensitive to the surroundings and the parasitics of the interconnection will affect the performance dramatically. The matching design and parasitics modeling are not mentioned in [11]. Also, extra interconnect would add more loss. For example, $0.8 \mathrm{~dB}$ loss is added due to transferring channel [12].

Wire-bonding is an interconnect technology that is widely used in (mm-wave) system packaging solutions. By designing matching network to compensate for the parasitic inductance of connecting wires, a good interconnect between chip and printed circuit board (PCB) can be obtained [14]-[23]. However, the performance is relatively narrow band and lossy. [15] proposed a dc-84 GHz interconnect by introducing an impedance transition on the chip. In this method, extra chip area is occupied and excessive grounded wires are used. The self-resonance frequency of the bondwire is also not mentioned. [18] and [23] proposed a new L-C-L structure to compensate for the bondwire influence. This design has the potential to achieve wideband interconnect above $100 \mathrm{GHz}$, but extra matching wires have to be used. [19] and [20] demonstrated a bondwire interconnection with wide-band (67$110 \mathrm{GHz}$ ) and low loss performance. The chip is placed into a cavity to reduce the wire length and characteristic impedance of the transmission line is chosen as $83 \Omega$ to improve the matching. On the other hand, flip-chip technology is an alternative option that has proven itself as the most promising solution for compact, low loss and broadband packaging technology [24]-[27]. The parasitic effect is much smaller than bondwires. Characterization of flip-chip interconnect has been verified at $120 \mathrm{GHz}$ in [25]. [27] proposed a high impedance coplanar waveguide (CPW) matching network for W-band applications.

Another important issue is wide band transmission line (TL) to rectangular waveguide transition (RWG). RWG is an important signal carrier for millimeter-wave system due to its low loss, simple topology, and high power-handing capability. It has been chosen as the type of testing ports of most of the 


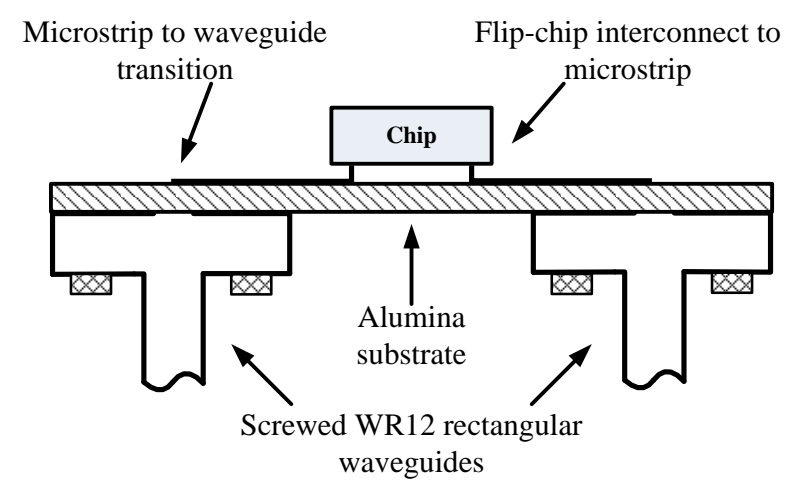

Fig. 1. Cross-section view of the proposed millimeter-wave packaging design from chip to WR-12 rectangular waveguide

measurement equipment. A good transition enables easy measurement set up for device testing. More importantly, in E-band communication systems, the antennas used in both transmitter and receiver sides should have a gain around $50 \mathrm{dBi}$ (minimum $43 \mathrm{dBi}$ ) to overcome the attenuation while extending the link distance and minimize the interference to other users [6]. To meet the gain requirement, reflector antennas with a waveguide feed port are typically used. Therefore, a good TL to RWG transition is needed. Recently, various RWG transitions have been published in the literature. A V-band substrate integrated waveguide (SIW) to RWG transition with more than $35 \%$ bandwidth (BW) is reported in [28]. SIW is a competitive planar signal carrier in (mm-wave) applications as the smaller dimension enables compact system integration. Also, it avoids using very thin metal lines and electromagnetic interference (EMI) problem because the signal is shielded. Since SIW is substrate-filled waveguide and realized with PCB technology, given a fixed substrate thickness, it is difficult to tune its impedance and low loss material should be used. [29] proposed a vertical transition from coupled microstrip line to RWG with 14.5 GHz bandwidth (center frequency $96 \mathrm{GHz}$ ). [19] and [20] presented a $67-110 \mathrm{GHz} \mathrm{CPW}$ to RWG transition. [30] presented a W-band LTCC transition from microstrip line to RWG. [31] presented a microstrip line to RWG transition with $15 \%$ bandwidth in E-band. These transitions show excellent performance within the working frequency range. However, either multiple-layer process or modified waveguide flanges, or back-short cavity have to be used. Additional fabrication errors are inevitable due to complex installation.

In this paper, a flip-chip packaging design from bond-pad to standard rectangular waveguide (WR-12) port is proposed for E-band long-haul communication systems. This work is an expansion of [21]. The cross-section view of the architecture is shown in Fig. 1. The proposed packaging is realized on a single-layer alumina board. The first part is a broadband flipchip interconnect to microstrip line. The on-chip G-S-G pads have the same $75 \mu \mathrm{m}$ standard pitch as the $110 \mathrm{GHz}$ probe, so the chip characteristics could be measured with probe station before packaging. The second part is microstrip line to WR12 standard RWG transition. The working frequency range is improved to cover the entire $71-86 \mathrm{GHz}$ bandwidth by suppressing higher order mode. There is no back-short cavity or modified waveguide involved. At last, a 40-nm CMOS Eband power amplifier (PA) assembled with the proposed design is measured. The measurements agree well with simulation, which verifies the validity of the design. Including loss of 10 $\mathrm{mm}$ micristrip line, from chip side to waveguide output port, measurement shows an insertion loss of $2.5 \mathrm{~dB}$, which satisfies the packaging loss requirement and leaves $5 \mathrm{~dB}$ margins for transmitter and receiver antenna alignment loss [6].

The paper is arranged as follows: The flip-chip to microstrip interconnect analysis is in Section II. The microstrip line to standard WR-12 waveguide (WG) transition and bandwidth enhancement design are discussed in Section III. Measurement results of a packaged E-band power amplifier are presented in Section IV. Comparison between flip-chip and bondwire packaging is discussed in Section V. A final conclusion is given in Section VI.

\section{CHIP PACKAGING WITH FLIP-CHIP INTERCONNECT}

\section{A. Flip-Chip to Microstrip Interconnect}

Compared with bondwire interconnect, flip-chip is more promising thanks to its smaller parasitic effects. An intuitive sense is that the width and spacing of the PCB trace is limited by the pad dimension. As the work in [32], a good interconnect can be realized if the impedance of the PCB transmission line and pad impedance are close to each other $(50 \Omega)$. However, the liquid crystal polymer (LCP) substrate thickness in [32] has to be reduced down to $50 \mu \mathrm{m}$ to satisfy both conditions at the same time. Although a good matching is achieved, the sample is soft and vulnerable. In other cases, the first step is to estimate the parasitic elements of the bumps and extract the equivalent circuit for further matching. Secondly, according to the moving track of the impedance at the target frequencies, design a matching network to bring the impedance point back to the center $(50 \Omega)$ of smith chart, which usually could be achieved by using a certain length of high impedance transmission line [26], [27].

In this design, the alumina is chosen to be carrier substrate based on the following considerations: 1) standard fabrication process. 2) mechanical strength and thermal stability to endure flip-chip process. 3) excellent performance at high frequencies. The thickness, permittivity and loss tangent of alumina substrate are $254 \mu \mathrm{m}, 9.5$ and 0.004 at $80 \mathrm{GHz}$, respectively. These would enable the width and gap of $50 \mathrm{CPW}$ line close to the pad dimensions, which have the size of $40 \mu \mathrm{m}$ by 50 $\mu \mathrm{m}$ and a standard pitch of $75 \mu \mathrm{m}$. The flip-chip interconnect design is performed in High Frequency Structure Simulator (HFSS), as shown in Fig. 2. The chip is replaced by $300 \mu \mathrm{m}$ thick silicon and the active circuit on the chip is replaced by a lumped port, which is connected to the G-S-G pads with the help of a short perfect conductor. The ground beneath the chip is removed to avoid an undesired impact on the on-chip passive components. Grouped metal-filled vias on each side connect the top and bottom ground together. These vias are placed as such to form a wideband transition from grounded CPW to microstrip line and also enhance the heat dissipation to the bottom metal ground. The microstrip line output goes into the 


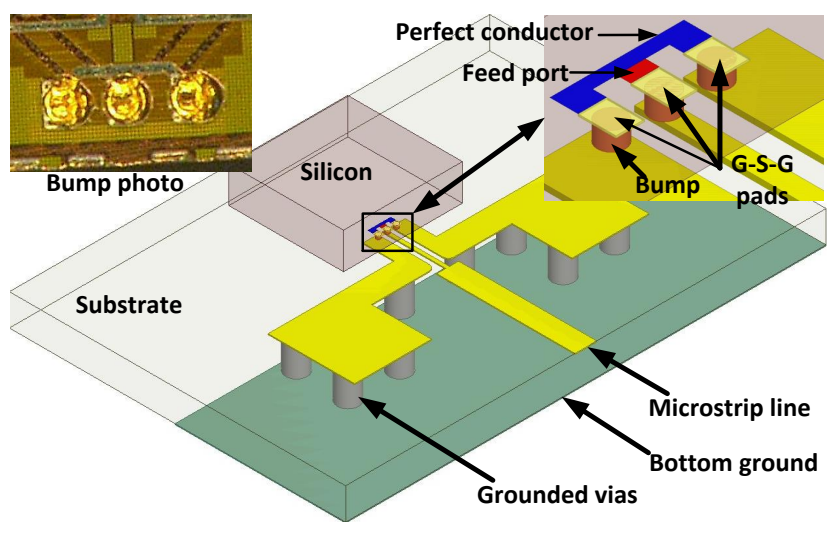

Fig. 2. Simulation model of flip-chip interconnect in HFSS and gold bump photo before flipping.

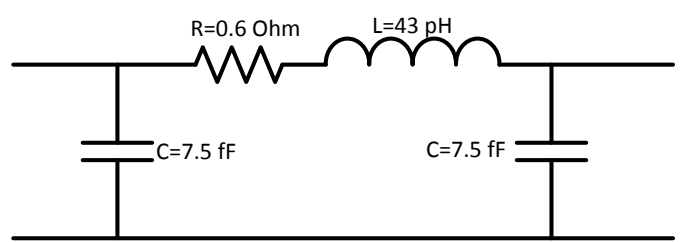

Fig. 3. Equivalent circuit of flip-chip bumps.

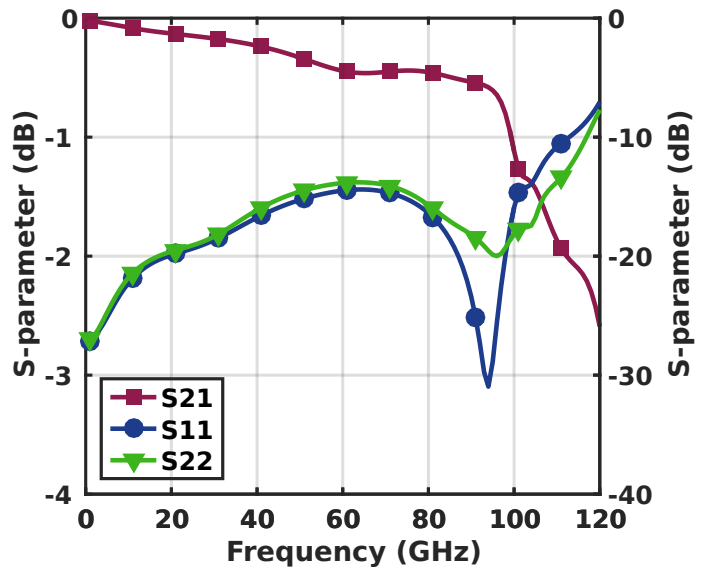

Fig. 4. Simulated S-parameters of flip-chip interconnect from on-chip G-S-G bond pads to microstrip line.

waveguide transition. The height and radius of gold bumps are both set as $20 \mu \mathrm{m}$ and $30 \mu \mathrm{m}$ in simulation. Equivalent cicuit of the bump interconnect is shown in Fig. 3. Because the characteristic impedance of both bond pad and grounded CPW line is $50 \Omega$, a good interconnect is achieved without compensation network [32]. The resonant peak between 80 $\mathrm{GHz}$ and $100 \mathrm{GHz}$ is due to grounded CPW to microstrip line transition. Simulated S-parameters are shown in Fig. 4. Return loss is lower than $-14 \mathrm{~dB}$ up to $100 \mathrm{GHz}$ and insertion loss is $0.5 \mathrm{~dB}$ at $77 \mathrm{GHz}$.

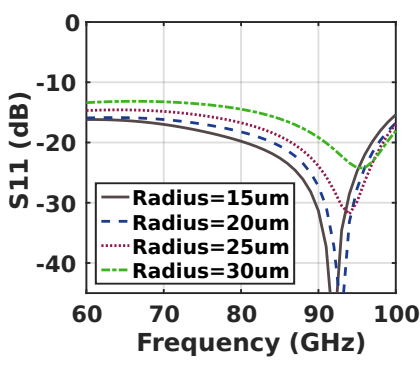

(a)

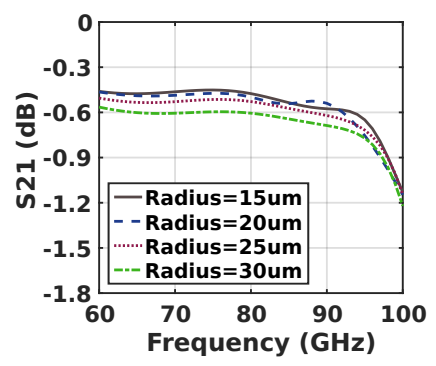

(b)
Fig. 5. Performance against bump radius variation when bump height is set as $20 \mu \mathrm{m}$. (a) $\mathrm{S} 11$, (b) $\mathrm{S} 21$.

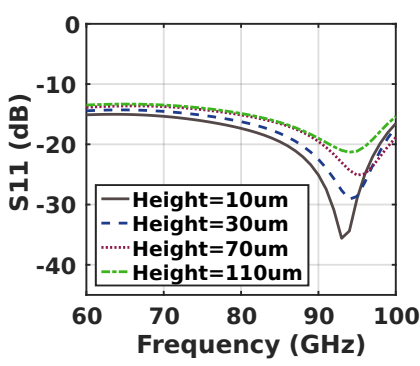

(a)

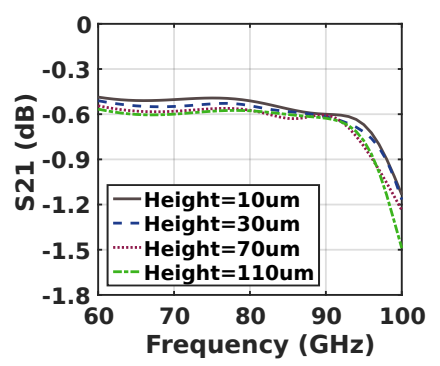

(b)
Fig. 6. Performance against bump height variation when bump radius is set as $20 \mu \mathrm{m}$. (a) S11, (b) S21.

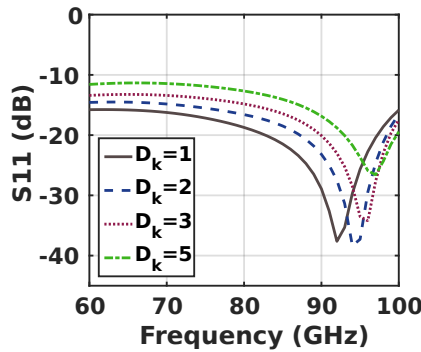

(a)

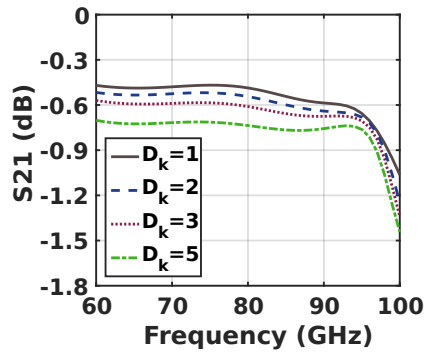

(b)
Fig. 7. Performance against different underfill materials. (a) S11, (b) S21.

\section{B. Parametric analysis}

To analyze the effect of the bump variation to the interconnect performance, the dimensions of the G-S-G bumps are swept in simulation. The radius and height are both set as 20 $\mu \mathrm{m}$, then keep one constant and sweep the other one. The results are shown in Fig. 5 and Fig. 6. It is clear that the performance is improved when the radius and height decrease. Similar result is achieved with [32]. In this design the pad size is $40 \mu \mathrm{m}$ by $50 \mu \mathrm{m}$ with $75 \mu \mathrm{m}$ pitch $(70 \mu \mathrm{m}$ by $70 \mu \mathrm{m}$ with $90 \mu \mathrm{m}$ pitch in [32]). Since the signal-ground gap is larger and the pad is smaller, capacitance effect is small and overruled by the inductance effect.

Fig. 7 shows the simulated S-parameters with different underfill materials. The main influence of using different underfill materials in flip-chip design is changing the equivalent permittivity $\left(\mathrm{D}_{k}\right)$ of the environment [33]. The impedance of the transmission line will be changed accordingly. As seen 


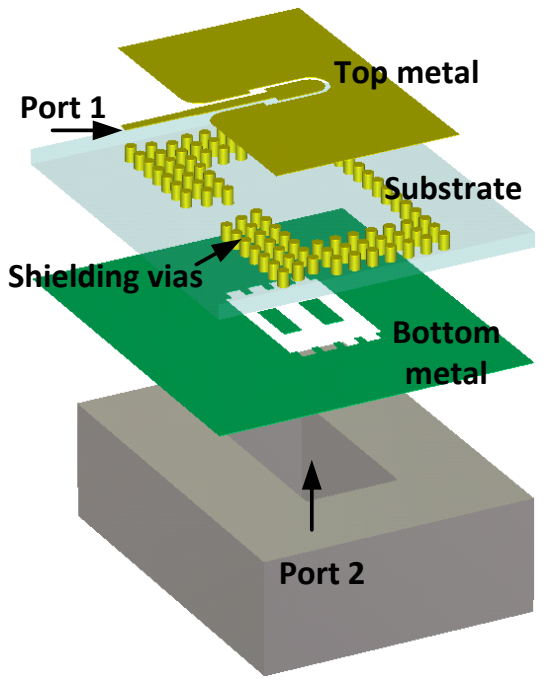

(a)
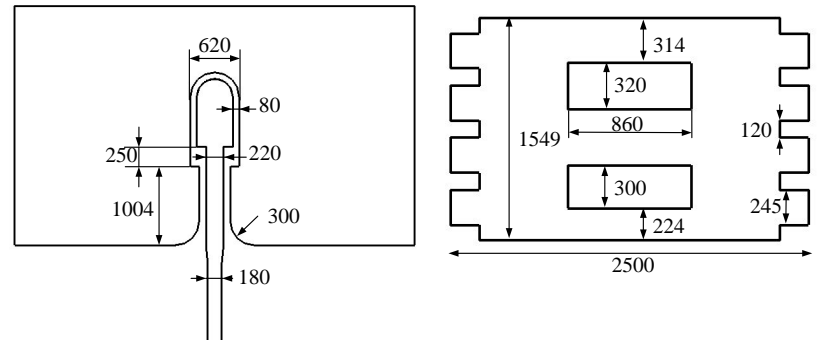

(b)

Fig. 8. Geometry of the proposed microstrip to RWG transition on alumina board: (a) overview, (b) parameters, unit in $\mu \mathrm{m}$.

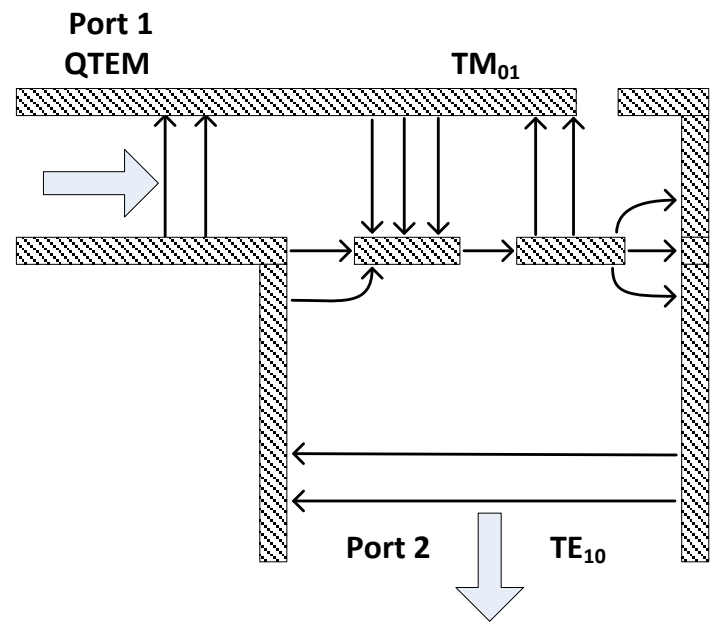

Fig. 9. Mode transformation from QTEM to $\mathrm{TE}_{10}$ mode.

from the result, better performance is achieved with low permittivity.

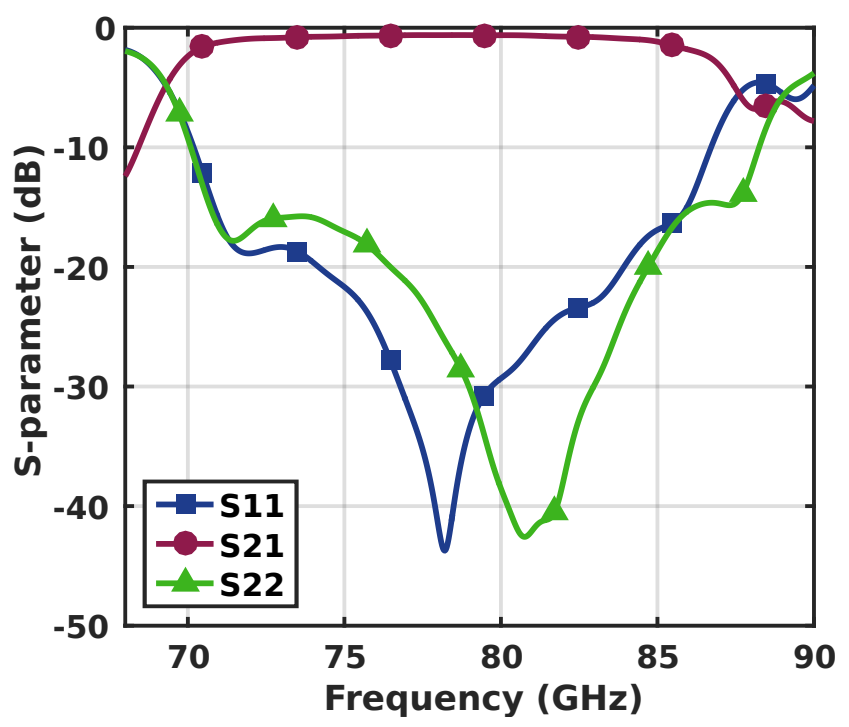

Fig. 10. Simulated S-parameters of the microstrip line to RWG transition.

\section{Microstrip to Waveguide Transition}

\section{A. Simulation model and mode analysis}

The proposed single-layer microstrip line to WR-12 waveguide transition is shown in Fig. 8(a) and the dimensions are given in Fig. 8(b). Fig. 9 illustrates the mode transition from QTEM mode to $\mathrm{TE}_{10}$ mode. The microstrip line connecting to the flip-chip packaging is located on the top metal layer of the alumina substrate. Two coupling patch elements are placed at the bottom metal layer facing to the waveguide, which is mounted to the substrate by screws. Metalized vias are placed around transition area to connect top and bottom grounds and form a good shielding. As shown in Fig. 10, the simulated return loss is better than $-15 \mathrm{~dB}$ from $71 \mathrm{GHz}$ to $86 \mathrm{GHz}$. Since there is no back-short cavity to shield the signal, the backside radiation leakage might be an issue to deteriorate the transition performance. The radiation loss is simulated when signal is fed from the microstrip port and waveguide port. As shown in Fig. 11, within the targeted frequency range only around 5\% of the signals get radiated out. It also means that the transition is practically not affected by any metal appearing behind the PCB. From $70.15 \mathrm{GHz}$ to $87.2 \mathrm{GHz}$, the average insertion loss is less than $0.5 \mathrm{~dB}$.

Traditional design for waveguide transition on single-layer substrate is using a rectangular patch element facing to the waveguide [34]. The signals get coupled from top CPW to bottom patch. Between this patch and rest of the ground, two slots are formed to radiate into waveguide. Unfortunately, this method is not suitable for transition design on substrate with high permittivity. Due to high permittivity property, the substrate wavelength becomes much shorter $(1.22 \mathrm{~mm}$ at 80 $\mathrm{GHz}$ ). The phase of E-field in the two slots are reversed to each other, thus instead of $\mathrm{TE}_{10}$ mode, $\mathrm{TM}$ mode will be excited. Since TM mode has a cut off frequency higher than $90 \mathrm{GHz}$, strong reflection occurs to the microstrip line port. To take the advantage of the short wavelength in the alumina substrate, in the proposed design, the rectangular patch is split 


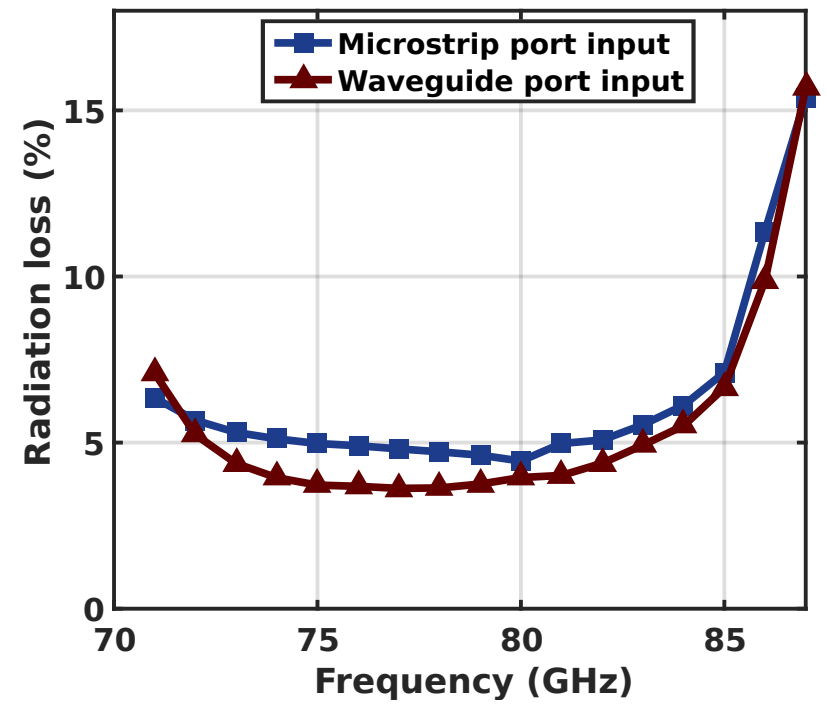

Fig. 11. Backside radiation loss of the proposed transition.

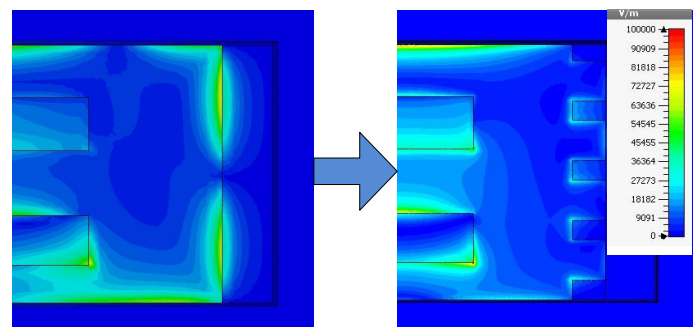

(a)

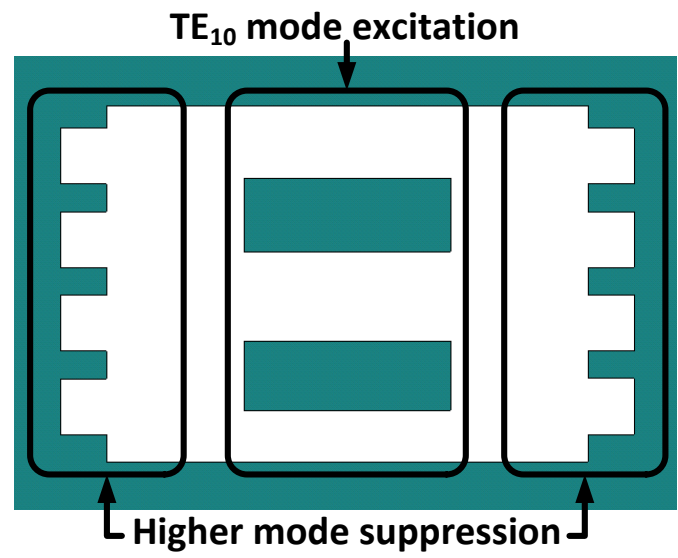

(b)

Fig. 12. Bandwidth enhancement: (a) transition interface, (b) E-field distribution comparison at $71 \mathrm{GHz}$.

into two parts. One extra slot is introduced into the interface between the two patches. By sizing the two patches, this new slot creates proper phase shift between the other two slots and thus the E-fields of these three slots are in phase. Therefore, the fundamental $\mathrm{TE}_{10}$ mode is excited into the waveguide. The design procedure is as follows: 1) Set the width of these three slots constant; 2) Aiming the center frequencies at 77

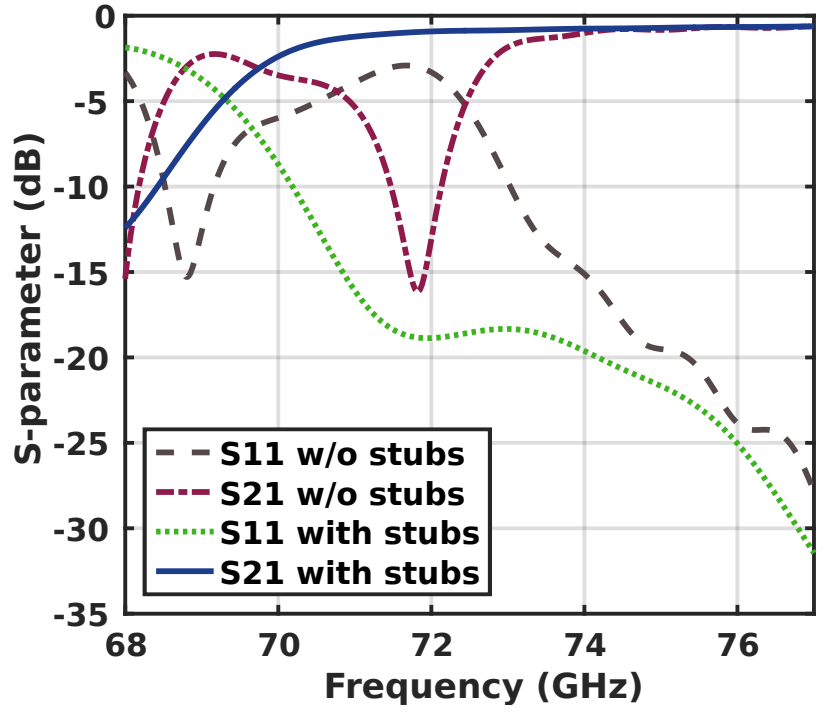

Fig. 13. Comparison of simulated S-parameters of bandwidth improvement.

$\mathrm{GHz}$, move the position of the middle slot, i.e. sweep the width of the two patches; 3) Finally optimize the length of the two patches. Simulation shows that better performance is achieved when the middle slot width is larger than the other two. It should be mentioned that although a rectangular slot is a resonant structure with narrow working bandwidth, the combination of the three slots enables a wide band operation in this design.

\section{B. Bandwidth Optimization}

The initial model shows the lower limit of working frequency is $73.3 \mathrm{GHz}$, which is not adequate for the application. In order to realize a waveguide transition which covers the entire 71 to $86 \mathrm{GHz}$, the magnitude distributions of the Efield at the waveguide interface is studied, as shown in Fig. 12. As can be seen in the left plot of Fig. 12(a), $\mathrm{TE}_{12}$ mode is excited at the interface, which could not propagate in WR-12 waveguide (cut off frequency higher than $90 \mathrm{GHz}$ ). A strong reflection thus occurs at the frequencies below $73.3 \mathrm{GHz}$. This phenomenon is a result of that the wavelength in the alumina substrate is much smaller. The size of the standing wave is smaller than the long side of the RWG, therefore, an induced traveling current occurs along the boundary. The $\mathrm{TE}_{12}$ mode can be compressed by shrinking the dimension of the transition interface and blocking its induced current on the boundary. The improved waveguide interface is shown in Fig. 12(b). Firstly, the long boundary of the interface is shortened from standard $3.988 \mathrm{~mm}$ to $2.5 \mathrm{~mm}$ to reduce the electrical length of the interface and to form a good standing wave. Then a line of choke slots (teeth) are placed along the two short boundaries to further block the induced current. The second technique is a typical method used in planar tapered slot antenna design, which suffers from side lobe radiation due to the boundary current. By placing choke slots along the boundary, the traveling current is reduced [35]. The teeth dimension can be calculated according to operating frequency 


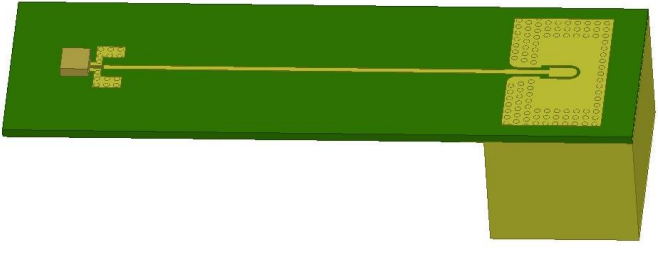

Fig. 14. Simulated model of G-S-G pads to RWG transition.

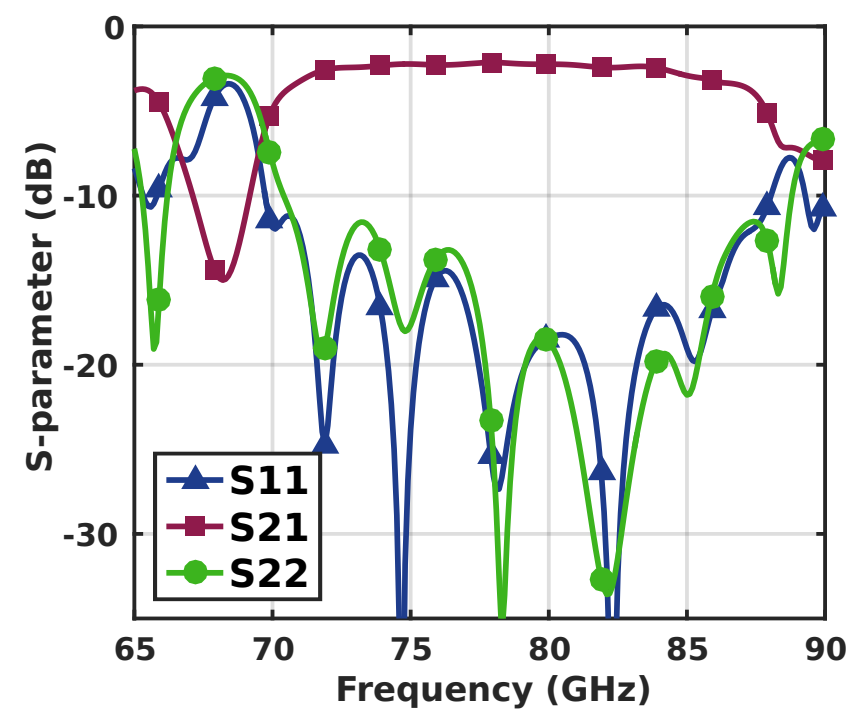

Fig. 15. Simulated S-parameters of G-S-G pads to RWG transition, including $12 \mathrm{~mm}$ microstrip line.

in antenna design. In this design, the dimension is swept and the optimized teeth width and slot width are chosen as $120 \mu \mathrm{m}$ and $245 \mu \mathrm{m}$. Fig. 12(b) shows the electrical field comparison with/without choke slots at $71 \mathrm{GHz}$.

The $\mathrm{TE}_{12}$ mode is compressed at the boundaries and the fundamental mode is formed by the coupling patches. The simulated S-parameters of the transition before and after modifying the coupling interface are presented in Fig. 13. As can be seen the lower working frequency moves from 73.3 $\mathrm{GHz}$ to $70.15 \mathrm{GHz}$. The simulated S-parameters of the final design are shown in Fig. 10. A promising microstrip to WR12 waveguide transition covering the targeted frequency range from $70.15 \mathrm{GHz}$ to $87.2 \mathrm{GHz}$ is achieved. The insertion loss at $77 \mathrm{GHz}$ is less than $0.5 \mathrm{~dB}$.

The co-simulation model of the entire G-S-G pads to RWG transition is shown in Fig. 14. To keep the chip away from the actual waveguide flange (otherwise flange metal will affect the performance of the chip), $10 \mathrm{~mm}$ microstrip line is used. The simulated S-parameters are shown in Fig. 15. As can be seen, a G-S-G pads to WR-12 standard RWG transition is achieved from $70 \mathrm{GHz}$ to $87 \mathrm{GHz}$, with an average insertion loss of 2.5 $\mathrm{dB}$.
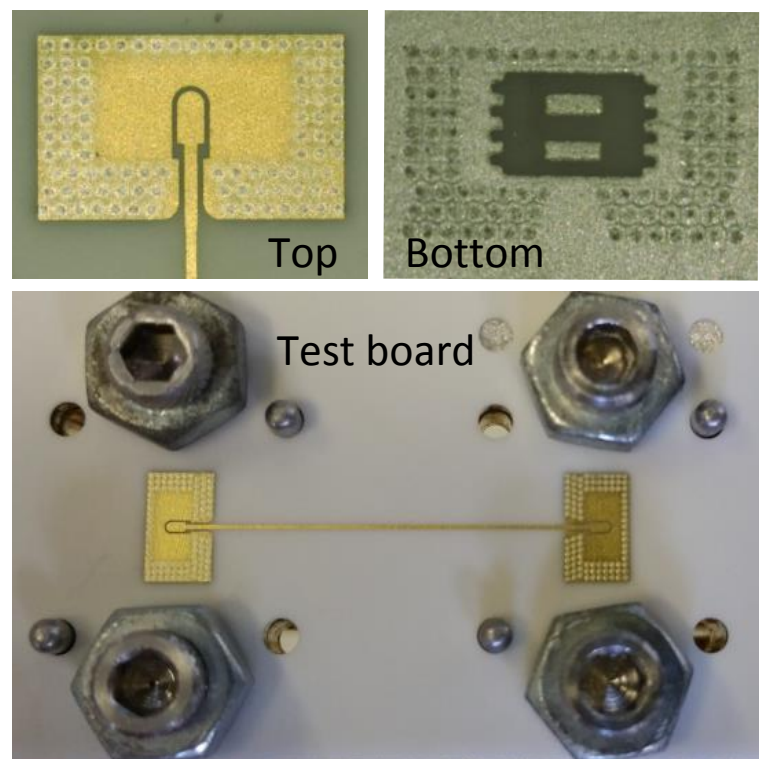

Fig. 16. Photograph of the fabricated back-to-back transition board.

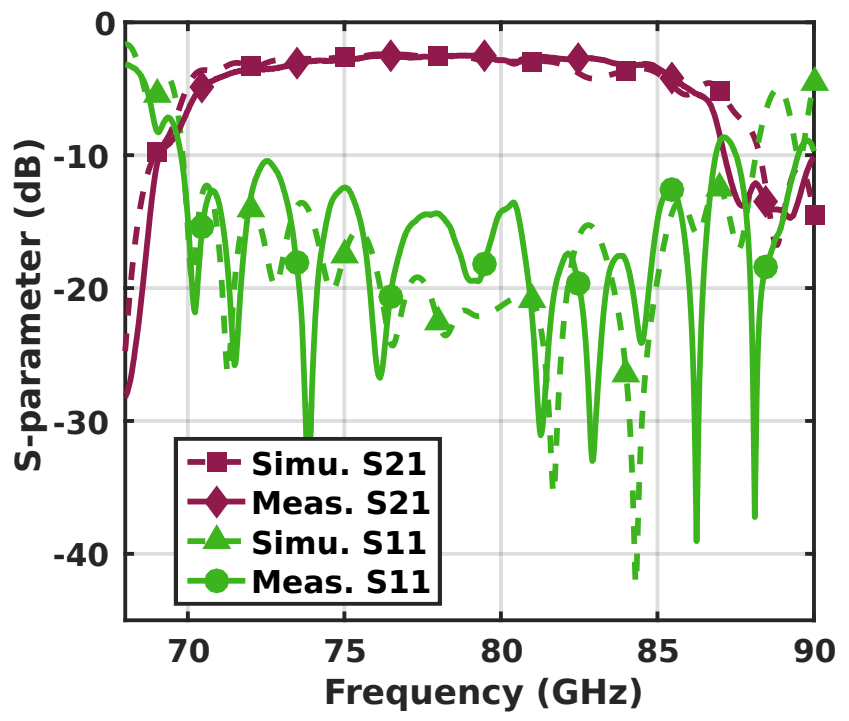

Fig. 17. Simulated and measured S-parameters of the back-to-back transition.

\section{FABRiCAtion And MeAsurement Result Analysis}

\section{A. Microstrip to WR-12 Waveguide Transition}

To verify the performance of the transition, a back-toback configuration (waveguide-to-waveguide) was fabricated and measured. Fig. 16 shows the photo of a back-to-back waveguide to microstrip transition board. The result will also be used for estimating the loss in the packaged power amplifier measurement. The S-parameter measurement was conducted using an R\&S vector network analyzer with E-band extenders. The measurement waveguide ports were screwed to the bottom of the board with the help of two 90 degree WR-12 bends. 
TABLE I

COMPARISON

\begin{tabular}{|l|c|c|c|c|c|}
\hline & {$[20]^{*}$} & {$[28]^{* *}$} & {$[29]$} & {$[30]$} & This work \\
\hline BW(GHz) & $60-110$ & $54-73.5$ & $88.7-103.3$ & $83-99$ & $69.5-87$ \\
\hline Loss(dB) & 0.35 & 0.58 & 0.5 & 0.7 & 0.5 \\
\hline Substrate & Rogers 5880 & Rogers 5880 & Taconic TLE-95 & LTCC & Alumina \\
\hline Modified WG & Yes & No & Yes & No & No \\
\hline Back-short & Yes & No & No & No & No \\
\hline Multi-layer & No & Yes & No & Yes & No \\
\hline $\begin{array}{l}* 83 \Omega \text { CPW line is used. } \\
* * \text { Estimated from plot. }\end{array}$
\end{tabular}

A waveguide TOSM (through-open-short-match) calibration was performed to the output port of the bends. Simulated and measured S-parameters are presented by dash and solid lines in Fig. 17. Measured result indicates that the proposed transition has return loss better than $10 \mathrm{~dB}$ from 69.5 to $87 \mathrm{GHz}$. Both simulation and measurement results show the insertion loss is $2.5 \mathrm{~dB}$ at $77 \mathrm{GHz}$. Since the simulated transmission loss of the $20 \mathrm{~mm}$ microstrip between two transitions is $1.7 \mathrm{~dB}$, the insertion loss of a single transition should be better than $0.5 \mathrm{~dB}$. The proposed design utilizes standard waveguide and expensive modified WG components or backshort cavity is avoided, therefore, the cost is reduced. In the meantime, minimization of fabrication errors and complexity are achieved. Table I gives a performance comparison of waveguide transitions among the literature.

\section{B. Packaged CMOS Power Amplifier}

A $40 \mathrm{~nm}$ CMOS power amplifier is packaged and measured with the proposed design. The gold bumps were placed on the pads, and then the chip was flipped onto the substrate. During the flip-chip process, the chip was pressed with 3 Newton $(\mathrm{N})$ force for $300 \mathrm{~ms}$ and the operating temperature is $80{ }^{\circ} \mathrm{C}$. The chip was glued to the board for protection using EPO-TEK H54 (permittivity 3.21, loss tangent 0.003 at $1 \mathrm{KHz}$ ) and there is no underfill material beneath the chip. After pressing the chip for adhesion, bump radius is around $30 \mu \mathrm{m}$, leaving only $10 \mu \mathrm{m}$ gap between each other. To avoid short connection, two ground bumps were placed deviating the pad center and the metal trace on PCB was therefore extended. By doing this the bump parasitic capacitance was increased [32]. To ensure the matching, the impedance of the CPW line is correspondingly increased [26]. S-parameters and temperature measurement setup are shown in Fig. 18. The photo of a flip-chip packaged E-band CMOS power amplifier board is shown in Fig. 19. The power amplifier has a supply voltage of $0.9 \mathrm{~V}$. The simulated and measured Sparameter results are presented in Fig. 20. Simulation result was obtained by combining S-matrixes of the chip and two G-S-G to WG transitions in Agilent Advanced Design System (ADS). Excellent agreement is achieved between simulated and measured results. A good matching is obtained within 71-86 GHz range and the $\mathrm{S} 12$ is lower than $-30 \mathrm{~dB}$ in the

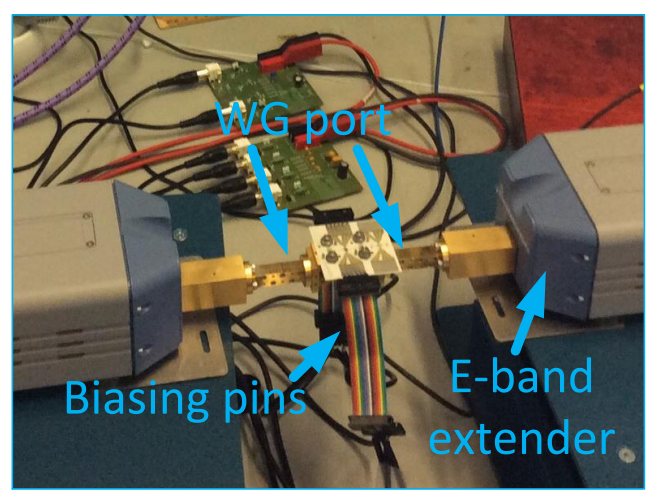

(a)

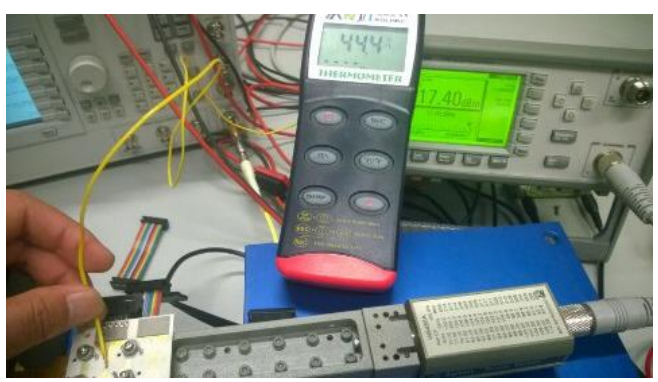

(b)

Fig. 18. Packaged PA measurement. (a) S-parameter measurement, (b) temperature measurement.

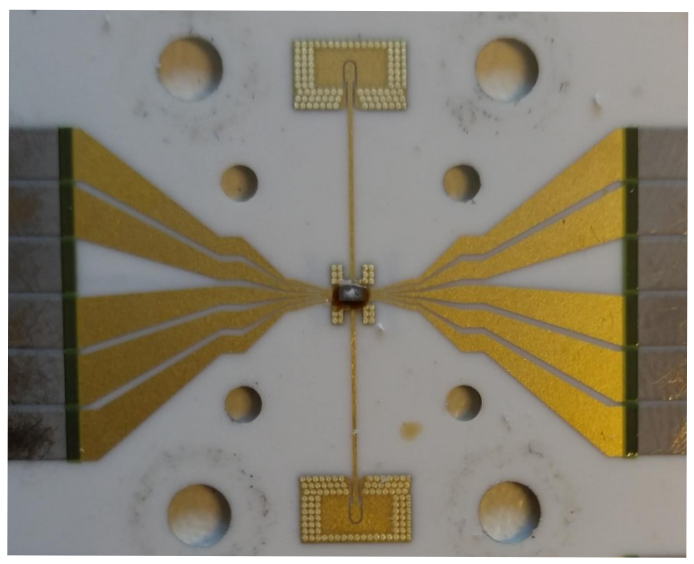

Fig. 19. Photograph of flip-chip packaged PA with waveguide transition.

entire E-band. Probe measurement shows the chip has a peak gain of $18.1 \mathrm{~dB}$ and packaging measurement achieves 13.4 $\mathrm{dB}$. Considering the loss of two flip-chip interconnects, $20 \mathrm{~mm}$ microstrip line and two waveguide transitions, the difference is less than $1 \mathrm{~dB}$. The output power is measured using the Aglient E4418B power meter. The measured peak power versus frequency is presented in Fig. 21. The maximum output power is $17.6 \mathrm{dBm}$ at $75 \mathrm{GHz}$. Since the chip performance is strongly affected by the increasing temperature, and to test the dissipation performance of the packaging, the packaged power 


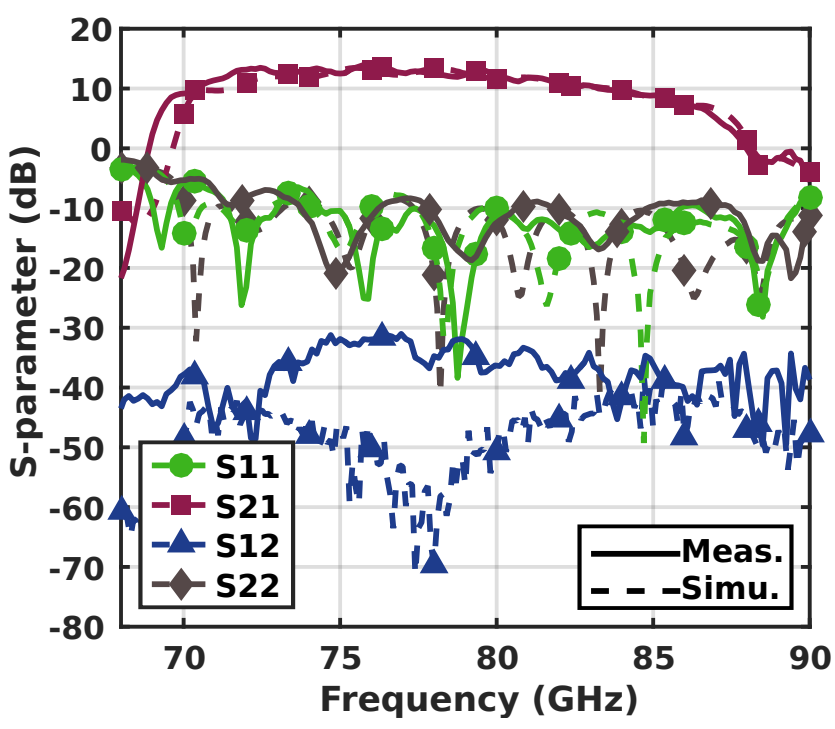

Fig. 20. Simulated and measured S-parameters of the packaged PA.

amplifier was set under saturation output condition, $77 \mathrm{GHz}$, in a $25^{\circ} \mathrm{C}$ environment for 30 minutes. A sensor is placed on top of the silicon substrate to collect the temperature information. The output power and consuming current keep stable during the entire test time. The temperature on the silicon surface is measured as $44.4{ }^{\circ} \mathrm{C}$.

\section{COMParison With Bondwire PaCKaging}

A bondwire packaging design using the same CMOS power amplifier chip was presented in [21]. The chip was placed into a cavity on the board to reduce the wire length. The equivalent inductance of the bondwire interconnect is calculated and compensated with transmission lines. Although the wire length is carefully controlled, its still a bit longer than expected. Gain and peak output power comparisons are shown in Fig. 21 and Fig. 22, respectively. Sample photos of the bondwire and flipchip interconnects with the same chip are presented in Fig. 23. The bondwire packaging achieves peak gain and output power of $11.7 \mathrm{~dB}$ and $16.3 \mathrm{dBm}$, respectively in measurement. According to the measurement results, bondwire interconnection shows a good performance and the wire-bonding packaging performance is mainly limited by the waveguide transition. In this work, the waveguide transition is optimized to achieve good performance from $69.5 \mathrm{GHz}$ to $87 \mathrm{GHz}$. Both flipchip and bondwire interconnects are verified to be valid in millimeter-wave packaging design.

As frequency goes higher, the parasitic element of the connection becomes dominant and will significantly impact the system performance. In (mm-wave) bondwire packaging design, the wire length is preferred to be reduced, usually by eliminating the height difference between chip and substrate [20]. Although the equivalent inductance of the bondwire can be modeled and calculated, its still not sufficient to support the interconnect design. Firstly, wire landing point on the substrate can be accurately controlled, but the actual wire length still suffers from fabrication inconsistency. Secondly,

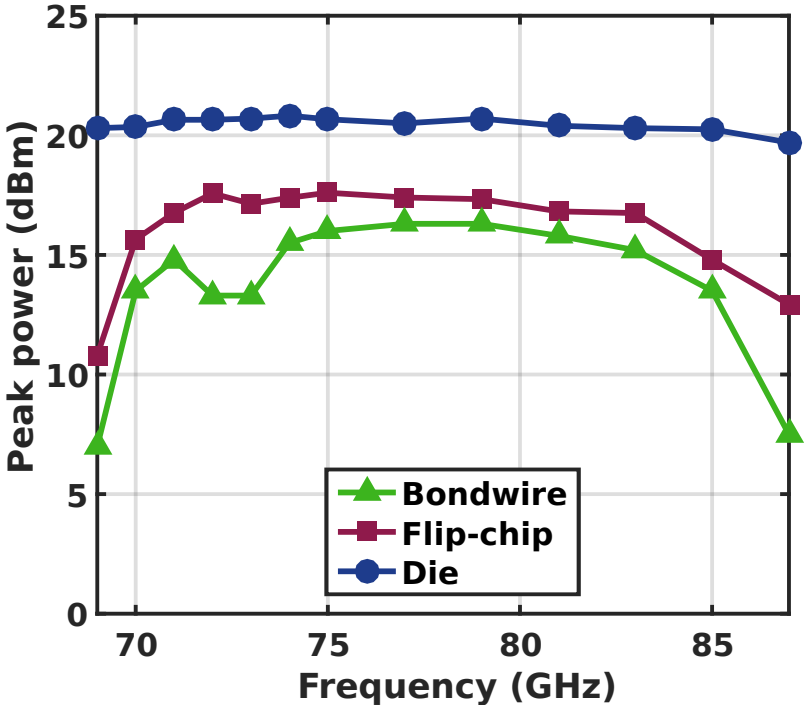

Fig. 21. Comparison of measured peak output power among bare die, bondwire packaging and flip-chip packaging [6], [21].

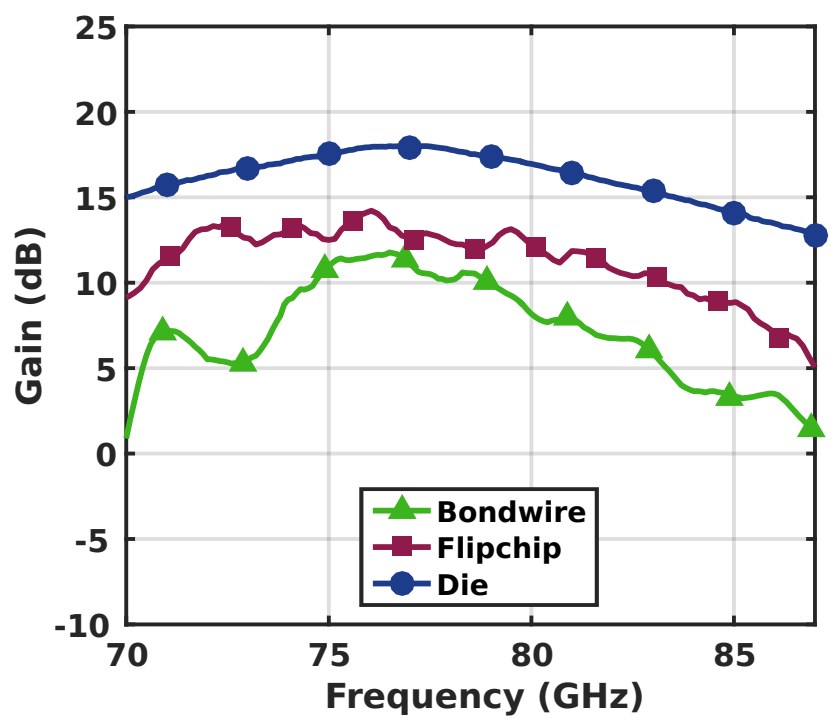

Fig. 22. Comparison of gain performance among bare die, bondwire packaging and flip-chip packaging [6], [21].

PCB details should be included in simulation environment. Ground terminal of the transmission line and via distribution, have severe influence to the practical wire model. Therefore, a set of simulations should be run to enhance the design stability. Another issue is that the wire self-resonant frequency should be away from the target frequency range, or a more complex model should be provided. To design a good flip-chip packaging there are some matters that need to be considered: Since the output power is large, input and output RF grounds need to be separated from each other to avoid feedback current and thus the circuit stability is improved. Remove the metal below the chip to avoid detuning effect of the on-chip passive components. The overlap between the pads and connecting trace needs to be minimized to reduce parasitic capacitance. 


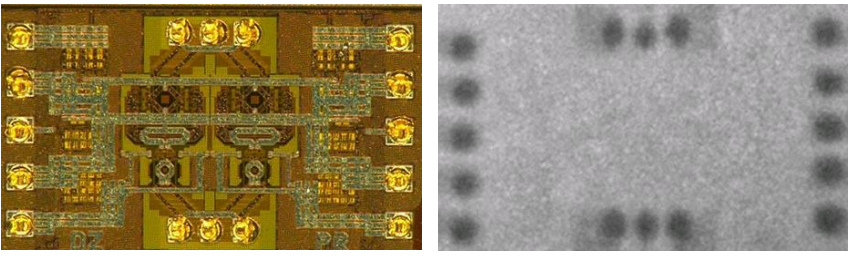

(a)

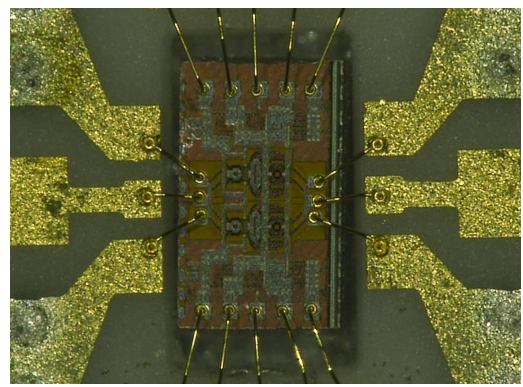

(b)

Fig. 23. Photograph of packaged chip: (a) chip with bumps before packaging (left), X-ray photo of packaged PA (right), (c) bondwire interconnect in [21].

Smaller connecting bumps are preferred to achieved better performance and interconnect design should be able to endure bump size variation. Carrier substrate is preferred to enable the impedance of the connecting trace close to $50 \Omega$, and have good thermal stability to endure the flip-chip process.

\section{CONCLUSION}

A flip-chip packaging design with rectangular waveguide output port has been implemented on single-layer alumina substrate, which is suitable for E-band long-haul communications. A broadband flip-chip interconnect is designed with standard $75 \mu \mathrm{m}$ pitch. To satisfy application requirement, single-layer microstrip line to WR-12 rectangular waveguide transition is theoretically and experimentally studied. Its working bandwidth covers from 69.5 to $87 \mathrm{GHz}$. By eliminating the transmission line loss, the insertion is less than $0.5 \mathrm{~dB}$ at $77 \mathrm{GHz}$. The packaging design utilizes standard process and components. The proposed solution is verified by measuring a $40 \mathrm{~nm}$ CMOS E-band power amplifier chip. Simulated and experimental results are in good agreement. The chip temperature is measured after operating at peak output state for 30 minutes. The packaged power amplifier achieves a peak gain and peak power of $13.4 \mathrm{~dB}$ and $17.6 \mathrm{dBm}$, respectively. After deembedding the loss, the measured result achieves similar performance with probe measurement. Finally, a comparison between flip-chip and bondwire packaging is discussed and both are verified in a millimeter-wave packaging design.

\section{ACKNOWLEDGMENT}

The authors would like to thank Frederik Daenen and Noella Gaethofs for their great support in the measurement.

\section{REFERENCES}

[1] S. Nicolson, P. Chevalier, B. Sautreuil, and S. Voinigescu, "Singlechip w-band sige hbt transceivers and receivers for doppler radar and millimeter-wave imaging," Solid-State Circuits, IEEE Journal of, vol. 43, no. 10, pp. 2206-2217, Oct 2008.

[2] D. Zhao, S. Kulkarni, and P. Reynaert, "A 60-ghz outphasing transmitter in 40-nm cmos," Solid-State Circuits, IEEE Journal of, vol. 47, no. 12, pp. 3172-3183, Dec 2012.

[3] P.-N. Chen, P.-J. Peng, C. Kao, Y.-L. Chen, and J. Lee, "A 94ghz 3dimage radar engine with $4 \mathrm{tx} / 4 \mathrm{rx}$ beamforming scan technique in $65 \mathrm{~nm}$ cmos," in Solid-State Circuits Conference Digest of Technical Papers (ISSCC), 2013 IEEE International, Feb 2013, pp. 146-147.

[4] J. Hasch, E. Topak, R. Schnabel, T. Zwick, R. Weigel, and C. Waldschmidt, "Millimeter-wave technology for automotive radar sensors in the $77 \mathrm{ghz}$ frequency band," Microwave Theory and Techniques, IEEE Transactions on, vol. 60, no. 3, pp. 845-860, March 2012.

[5] U. Pfeiffer and D. Goren, "A 23-dbm 60-ghz distributed active transformer in a silicon process technology," Microwave Theory and Techniques, IEEE Transactions on, vol. 55, no. 5, pp. 857-865, May 2007.

[6] D. Zhao and P. Reynaert, "An e-band power amplifier with broadband parallel-series power combiner in 40-nm cmos," Microwave Theory and Techniques, IEEE Transactions on, vol. 63, no. 2, pp. 683-690, Feb 2015.

[7] M. Khanpour, K. Tang, P. Garcia, and S. Voinigescu, "A wideband w-band receiver front-end in 65-nm cmos," Solid-State Circuits, IEEE Journal of, vol. 43, no. 8, pp. 1717-1730, Aug 2008.

[8] W. Chan and J. Long, "A 58-65 ghz neutralized cmos power amplifier with pae above 10vol. 45, no. 3, pp. 554-564, March 2010.

[9] T. Redant and W. Dehaene, "Joint estimation of propagation delay dispersion and time of arrival in a 40-nm cmos comparator bank for time-based receivers," Circuits and Systems II: Express Briefs, IEEE Transactions on, vol. 60, no. 2, pp. 76-80, Feb 2013.

[10] C. Doan, S. Emami, A. Niknejad, and R. Brodersen, "Millimeter-wave cmos design," Solid-State Circuits, IEEE Journal of, vol. 40, no. 1, pp. 144-155, Jan 2005.

[11] R. Li, C. Jin, S. C. Ong, T. G. Lim, K. F. Chang, and S. W. Ho, "Embedded wafer level packaging for 77-ghz automotive radar front-end with through silicon via and its 3-d integration," Components, Packaging and Manufacturing Technology, IEEE Transactions on, vol. 3, no. 9, pp. 1481-1488, Sept 2013.

[12] Y. Y. Lim, X. Xiao, S. Vempati, N. Su, A. Kumar, G. Sharma, T. G. Lim, K. Vaidyanathan, J. Shi, J. Lau, and S. Liu, "High quality and low loss millimeter wave passives demonstrated to 77-ghz for sip technologies using embedded wafer-level packaging platform (emwlp)," Advanced Packaging, IEEE Transactions on, vol. 33, no. 4, pp. 1061-1071, Nov 2010.

[13] M. Wojnowski, R. Lachner, J. Bock, C. Wagner, F. Starzer, G. Sommer, K. Pressel, and R. Weigel, "Embedded wafer level ball grid array (ewlb) technology for millimeter-wave applications," in Electronics Packaging Technology Conference (EPTC), 2011 IEEE 13th, Dec 2011, pp. 423429.

[14] J. Li, Y.-Z. Xiong, S. Hu, W. L. Goh, D. Hou, and W. Wu, "Performance analyse on millimetre-wave bonding-wire interconnection," in Electrical Design of Advanced Packaging Systems Symposium (EDAPS), 2010 IEEE, Dec 2010, pp. 1-4.

[15] C.-H. Li, C.-L. Ko, C.-N. Kuo, M.-C. Kuo, and D.-C. Chang, "A lowcost dc-to-84-ghz broadband bondwire interconnect for sop heterogeneous system integration," Microwave Theory and Techniques, IEEE Transactions on, vol. 61, no. 12, pp. 4345-4352, Dec 2013.

[16] T. Budka, "Wide-bandwidth millimeter-wave bond-wire interconnects," Microwave Theory and Techniques, IEEE Transactions on, vol. 49, no. 4, pp. 715-718, Apr 2001.

[17] T. Krems, W. Haydl, H. Massler, and J. Rudiger, "Millimeter-wave performance of chip interconnections using wire bonding and flip chip," in Microwave Symposium Digest, 1996., IEEE MTT-S International, vol. 1, June 1996, pp. 247-250 vol.1.

[18] G. Liu, A. Trasser, A. Ulusoy, and H. Schumacher, "Low-loss, low-cost, ic-to-board bondwire interconnects for millimeter-wave applications," in Microwave Symposium Digest (MTT), 2011 IEEE MTT-S International, June 2011, pp. 1-1.

[19] S. Jameson and E. Socher, "A 67-110ghz cmos to wr-10 waveguide transition using wirebonds and wideband microstrip launcher," in Microwave Symposium (IMS), 2014 IEEE MTT-S International, June 2014, pp. 1-4.

[20] $\_$, "A wide-band cmos to waveguide transition at mm-wave frequencies with wire-bonds," Microwave Theory and Techniques, IEEE Transactions on, vol. 63, no. 9, pp. 2741-2750, Sept 2015. 
[21] Y. Zhang, D. Zhao, and P. Reynaert, "Millimeter-wave packaging on alumina board for e-band cmos power amplifiers," in Power Amplifiers for Wireless and Radio Applications (PAWR), 2015 IEEE Topical Conference on, Jan 2015, pp. 1-3.

[22] T. Krems, W. Haydl, L. Verweyen, M. Schlechtweg, H. Massler, and J. Rudiger, "Coplanar bond wire interconnections for millimeter-wave applications," in Electrical Performance of Electronic Packaging, 1995, Oct 1995 , pp. $178-180$.

[23] V. Valenta, H. Schumacher, T. Spreng, V. Ziegler, D. Dancila, and A. Rydberg, "Experimental evaluation of differential chip-to-antenna bondwire interconnects above 110 ghz," in Microwave Conference (EuMC), 2014 44th European, Oct 2014, pp. 1008-1011.

[24] C.-C. Kuo, H.-C. Lu, P.-A. Lin, C.-F. Tai, Y.-M. Hsin, and H. Wang, "A fully sip integrated $\mathrm{v}$-band butler matrix end-fire beam-switching transmitter using flip-chip assembled cmos chips on ltcc," Microwave Theory and Techniques, IEEE Transactions on, vol. 60, no. 5, pp. 1424 1436, May 2012.

[25] S. Beer, H. Gulan, C. Rusch, and T. Zwick, "Integrated 122-ghz antenna on a flexible polyimide substrate with flip chip interconnect," Antennas and Propagation, IEEE Transactions on, vol. 61, no. 4, pp. 1564-1572, April 2013.

[26] A. Jentzsch and W. Heinrich, "Theory and measurements of flip-chip interconnects for frequencies up to $100 \mathrm{ghz}$," Microwave Theory and Techniques, IEEE Transactions on, vol. 49, no. 5, pp. 871-878, May 2001.

[27] H.-C. Lu, C.-C. Kuo, P.-A. Lin, C.-F. Tai, Y.-L. Chang, Y.-S. Jiang, J.-H. Tsai, Y.-M. Hsin, and H. Wang, "Flip-chip-assembled w -band cmos chip modules on ceramic integrated passive device with transition compensation for millimeter-wave system-in-package integration," $M i$ crowave Theory and Techniques, IEEE Transactions on, vol. 60, no. 3 , pp. 766-777, March 2012.

[28] Y. Li and K.-M. Luk, "A broadband v-band rectangular waveguide to substrate integrated waveguide transition," Microwave and Wireless Components Letters, IEEE, vol. 24, no. 9, pp. 590-592, Sept 2014.

[29] Z. Tong and A. Stelzer, "A vertical transition between rectangular waveguide and coupled microstrip lines," Microwave and Wireless Components Letters, IEEE, vol. 22, no. 5, pp. 251-253, May 2012.

[30] B. Cao, H. Wang, Y. Huang, J. Wang, and W. Sheng, "A w-band low-loss and wideband ltcc transition from waveguide to microstrip," Microwave and Wireless Components Letters, IEEE, vol. 23, no. 11, pp. 572-574, Nov 2013.

[31] E. Topak, J. Hasch, and T. Zwick, "Compact topside millimeter-wave waveguide-to-microstrip transitions," Microwave and Wireless Components Letters, IEEE, vol. 23, no. 12, pp. 641-643, Dec 2013.

[32] W. Khan, A. Lopez, A. Ulusoy, and J. Papapolymerou, "Packaging a w -band integrated module with an optimized flip-chip interconnect on an organic substrate," Microwave Theory and Techniques, IEEE Transactions on, vol. 62, no. 1, pp. 64-72, Jan 2014.

[33] Z. Feng, W. Zhang, B. Su, K. Gupta, and Y. Lee, "Rf and mechanical characterization of flip-chip interconnects in cpw circuits with underfill," Microwave Theory and Techniques, IEEE Transactions on, vol. 46, no. 12, pp. 2269-2275, Dec 1998.

[34] H. Iizuka, K. Sakakibara, and N. Kikuma, "Millimeter-wave transition from waveguide to two microstrip lines using rectangular patch element," Microwave Theory and Techniques, IEEE Transactions on, vol. 55, no. 5, pp. 899-905, May 2007.

[35] G. Teni, N. Zhang, J. Qiu, and P. Zhang, "Research on a novel miniaturized antipodal vivaldi antenna with improved radiation," Antennas and Wireless Propagation Letters, IEEE, vol. 12, pp. 417-420, 2013.

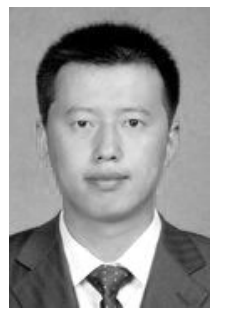

Yang Zhang (S'15) received the M.Sc. degree in electromagnetic field and microwave technology from Harbin Institute of Technology, Harbin, China, in 2013. He is currently working toward the Ph.D. degree at University of Leuven (KU Leuven), Leuven, Belgium. His current research interests include millimeter-wave CMOS circuits, packaging and interface design.

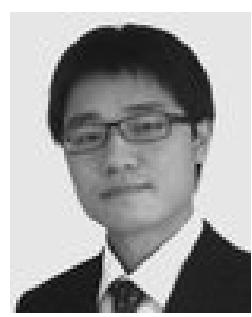

Dixian Zhao Dixian Zhao (S'10, M'15) received the B.Sc. degree in microelectronics from Fudan University, Shanghai, China, in 2006, the M.Sc. degree in microelectronics from Delft University of Technology (TU Delft), Delft, The Netherlands, in 2009, and the Ph.D. degree at University of Leuven (KU Leuven), Leuven, Belgium, in 2015. From late 2005 to 2007, he was with the AutoID Laboratory, Fudan University, Shanghai, China, where he developed nonvolatile memory for passive RFID tags. From 2008 to 2009, he was an Intern with Philips Research, Eindhoven, The Netherlands, where he designed a 60-GHz beamforming transmitter for presence detection radar. From 2009 to 2010 , he was with TU Delft, where he designed a $94-\mathrm{GHz}$ wideband receiver for imaging radar. From 2010 to 2015 , he was a research associate with KU Leuven, working on $60-\mathrm{GHz}$ and E-band transmitters and power amplfiers. Since April 2015, he has been an Associate Professor at Southeast University, China. His current research interests include RF and millimeterwave integrated transceiver and power amplifier for wireless communications. Dr. Zhao was the recipient of the IEEE Solid-State Circuits Society Predoctoral Achievement Award in 2014, the Chinese Government Award for Outstanding Students Abroad in 2013, the Top-Talent Scholarship from TU Delft in 2007 and 2008, and the Samsung Fellowship in 2005.

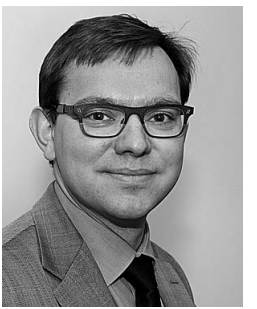

Patrick Reynaert (SM'11) was born in Wilrijk, Belgium, in 1976. He received the Master of Industrial Sciences in Electronics (ing.) from the Karel de Grote Hogeschool, Antwerpen, Belgium in 1998 and both the Master of Electrical Engineering (ir.) and the Ph.D. in Engineering Science (dr.) from the University of Leuven (KU Leuven), Belgium in 2001 and 2006 respectively. During 2006-2007, he was a post-doctoral researcher at the Department of Electrical Engineering and Computer Sciences of the University of California at Berkeley, with the support of a BAEF Francqui Fellowship. During the summer of 2007, he was a visiting researcher at Infineon, Villach, Austria. Since October 2007, he is a Professor at the University of Leuven (KU Leuven), department of Electrical Engineering (ESAT-MICAS). His main research interests include mm-wave and $\mathrm{THz}$ CMOS circuit design, high-speed circuits and RF power amplifiers. Patrick Reynaert is a Senior Member of the IEEE and chair of the IEEE SSCS Benelux Chapter. He serves or has served on the technical program committees of several international conferences including ISSCC, ESSCIRC, RFIC, PRIME and IEDM. He has served as Associate Editor for Transactions on Circuits and Systems I, and as Guest Editor for the Journal of Solid-State Circuits.

He received the 2011 TSMC-Europractice Innovation Award, the ESSCIRC2011 Best Paper award and the 2014 2nd Bell Labs Prize. 